\title{
Improving the effectiveness of routine prenatal screening for major congenital heart defects
}

\author{
J S Carvalho, E Mavrides, E A Shinebourne, S Campbell, B Thilaganathan
}

Heart 2002;88:387-391

See end of article for authors' affiliations

Correspondence to: Dr Julene S Carvalho, Royal Brompton Hospital, Sydney Street, London SW3 6NP, UK i.carvalho@

rbh.nthames.nhs.uk

Accepted 12 June 2002

\begin{abstract}
Objective: To evaluate the effectiveness of adding outlet views to the four chamber view in routine prenatal ultrasound screening for major congenital heart defects (CHD) as performed by trained sonographers, and to compare the procedure with current practice.

Design and setting: Prospective observational study at a London teaching hospital.

Participants and methods: 9277 women booked at a single institution $180 \%$ had first trimester nuchal translucency measurement) due to have routine fetal cardiac screening using the four chamber and outflow tract views at $>18$ weeks of gestation.

Main outcome measure: Identification of major CHD in chromosomally normal and abnormal pregnancies antenatally or postnatally.

Results: There were 40 abnormalities (4.3/1000), of which 30 were chromosomally normal (3.3/1000). The overall antenatal detection rate was $75 \%(95 \%$ confidence interval (CI) $59 \%$ to $87 \%)$ and $70 \%(95 \% \mathrm{Cl} 51 \%$ to $85 \%)$ for euploid pregnancies. Abnormal cardiac views accounted for $70 \%$ of all prenatal diagnoses, $30 \%$ of which were made at $\leqslant 18$ weeks. The sensitivity of cardiac views during the first scan at $>18$ weeks was $52 \%$. Of all patients undergoing nuchal translucency screening, 34 had major CHD, nine with increased nuchal translucency $(26.5 \%)$. Factors influencing the results of this screening programme were training and audit of operators, adequate equipment for antenatal examination, ease of access, and low threshold for referral to specialised fetal echocardiography.

Conclusion: Adding ventricular outlet views to the four chamber assessment of the heart at routine fetal anomaly scans at $>18$ weeks is the most effective technique to detect CHD prenatally. The success of such a programme depends on an infrastructure committed to continuous in house training of obstetric ultrasonographers coupled with feedback from specialised fetal cardiologists, as well as adequate resource allocation to obstetric hospitals involved with antenatal screening.
\end{abstract}

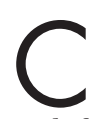
ongenital heart disease accounts for the majority of deaths from congenital defects in childhood, being six times more common than chromosomal abnormalities and four times more common than neural tube defects. Prenatal detection of specific cardiac anomalies such as complete transposition of the great arteries and hypoplastic left heart syndrome has been shown to improve neonatal morbidity and surgical outcome. ${ }^{12}$ The overall prevalence of congenital heart defects (CHD) is estimated at 8/1000 live births. ${ }^{3-5}$ Defects usually classified as major or critical are those that are lethal or require intervention in infancy or on long term follow up. The estimated prevalence of such major abnormalities is 4/1000 live births. ${ }^{6}$

Historically, detailed fetal echocardiography was performed because of a positive family history for CHD, but cardiac scanning has gradually been incorporated into routine ultrasound screening programmes without strong evidence to support its implementation. Over the years, reported sensitivities for major CHD by examination of the fetal four chamber view in low risk populations at $18-23$ weeks has been extremely variable, ranging form $5-60 \% .^{6-13}$ In the UK, the most recent national survey of over 4000 cases of major CHD reported an average antenatal detection rate of $23 \%{ }^{14}$

Adding visualisation of the ventricular outflow tracts to assessment of the four chamber view has been suggested as likely to increase the sensitivity of ultrasound screening for major CHD. ${ }^{15}{ }^{16}$ However, in most previous studies selected populations were examined by specially trained medical staff ${ }^{15}{ }^{16}$ who were allocated a longer time for scanning and incorporated colour flow Doppler. ${ }^{16}$ Recently, Hunter and colleagues ${ }^{17}$ instituted a policy of training sonographers involved in routine prenatal screening to obtain these views and showed an increase in detection rates. We have further developed such a programme with the aim of assessing the effectiveness of adding outlet views to the four chamber view in routine 18-23 week anomaly scans obtained by ultrasonographers.

\section{PATIENTS AND METHODS}

This was a prospective observational study of an unselected obstetric population in whom routine anomaly scans are performed at 18-23 weeks' gestation. Between January 1997 and August 1999 all women booked for antenatal care at our institution and subsequently delivered within our unit were included in the study. High risk referrals from other hospitals were excluded to eliminate bias.

Two full time and four part time sonographers working only in obstetric ultrasound performed routine ultrasound scans. The obstetric ultrasound service was situated within a unit that provided fetal medicine and echocardiography services. High resolution ultrasound equipment with a cineloop facility (ATL 3000/5000, Letchworth, UK and Acuson XP10, Uxbridge, UK) was used. In the majority of patients, nuchal translucency thickness was measured in the first trimester for screening of aneuploidy, as previously described. ${ }^{18}$ At the 18-23 week ultrasound scan, as part of the routine structural survey, the four chamber view of the fetal heart was examined. With the inception of fetal cardiology services within the unit, a comprehensive training programme overseen by the fetal cardiologist was instituted for all radiographers and obstetricians working in obstetric ultrasound.

The training was focused on two main principles. The first was that operators optimised the machine settings to obtain 
satisfactory cardiac images. The second was the use of a checklist for detailed assessment of the four chamber view. In addition, all sonographers were taught how to assess the outflow tracts and were encouraged to extend the echocardiographic views to include the great arteries in all patients. The time allocated for the entire fetal anomaly scan was 20 minutes per patient, with most sonographers spending an average of 2-3 minutes (maximum 5-6 minutes) to assess the fetal heart. Whenever an abnormality was detected or suspected at this level, patients were referred for a detailed cardiac scan by the fetal cardiologist. The training was complemented by two policies within the department to ensure ease of access to fetal cardiology. Firstly, in addition to those thought to be possibly or frankly abnormal, all cases with unsatisfactory views of the fetal heart were referred to the fetal cardiologist. Whenever possible the sonographer would observe the detailed cardiac scan to compare the findings with his or her own scan. Additionally, the sonographers were provided with a detailed explanation of the abnormalities encountered and were subsequently given feedback regarding postnatal outcome or postmortem results in terminated pregnancies.

\section{Referral for echocardiography \\ Prenatal}

Any deviation from the normal appearance of the fetal heart or an unsatisfactory (inability to establish normal anatomy) cardiac view was considered an indication for more detailed fetal echocardiography at any stage of pregnancy. In addition, all pregnancies considered to be at high risk of fetal cardiac problems were referred for detailed fetal echocardiography at 20-23 weeks. ${ }^{19-23}$ These included diabetic pregnancy, extracardiac fetal abnormalities detected on ultrasound, and a family history of CHD. Women with fetal nuchal translucency measurements above the 99th centile were also referred for early fetal echocardiography at 13-16 weeks. ${ }^{24-27}$

\section{Postnatal}

Echocardiograms were routinely recorded from neonates with signs of heart failure or cyanosis; when a heart murmur was associated with abnormal chest radiograph or ECG; or when a murmur persisted for more than six weeks in asymptomatic children. Additionally, all neonates in whom a cardiac anomaly was diagnosed in the antenatal period had postnatal echocardiography to confirm the diagnosis.

\section{Classification of CHD}

Cardiac defects were classified as major when they were potentially lethal or were severe enough to warrant termination of pregnancy, required surgery or interventional catheterisation in infancy, or were likely to need treatment on long term follow up. Patent ductus arteriosus, atrial septal defects (ostium secundum), small and restrictive ventricular septal defects, and valvar pulmonary stenosis with Doppler gradients $\leqslant 35 \mathrm{~mm} \mathrm{Hg}$ were classified as minor for the purposes of this study.

\section{Data collection}

Patient demographic, ultrasound, and pregnancy outcome data were recorded prospectively on a computer database. Chromosomal abnormalities were identified by reviewing all abnormal fetal and infant karyotypes from the regional cytogenetic laboratory database. In pregnancies with fetal cardiac malformations that resulted in intrauterine death or where the parents opted for termination of pregnancy, detailed pathological examination was requested to confirm the antenatal diagnosis. All neonatal and infant echocardiograms performed during the study period and up until April 2000 were reviewed to identify cardiac defects not diagnosed in the prenatal period. All cases of major CHD diagnosed in our institution are referred to a single cardiac centre. Neonatal, obstetric, and cardiothoracic teams met weekly to exchange information regarding recently diagnosed cardiac anomalies. The cardiothoracic database of the tertiary cardiac centre was also reviewed to identify patients from our unit undergoing long term follow up, interventional cardiac catheterisation, or surgery.

\section{RESULTS}

During the study period 9277 women booked for antenatal care delivered within the unit. The mean maternal age of the study population was 27 years (range 15-44 years). First trimester nuchal translucency was measured in 7384 (79.6\%) pregnancies.

\section{Referrals}

Referrals for echocardiography were made in 890 (9.6\%) pregnancies and 517 children. Figure 1 shows the indications

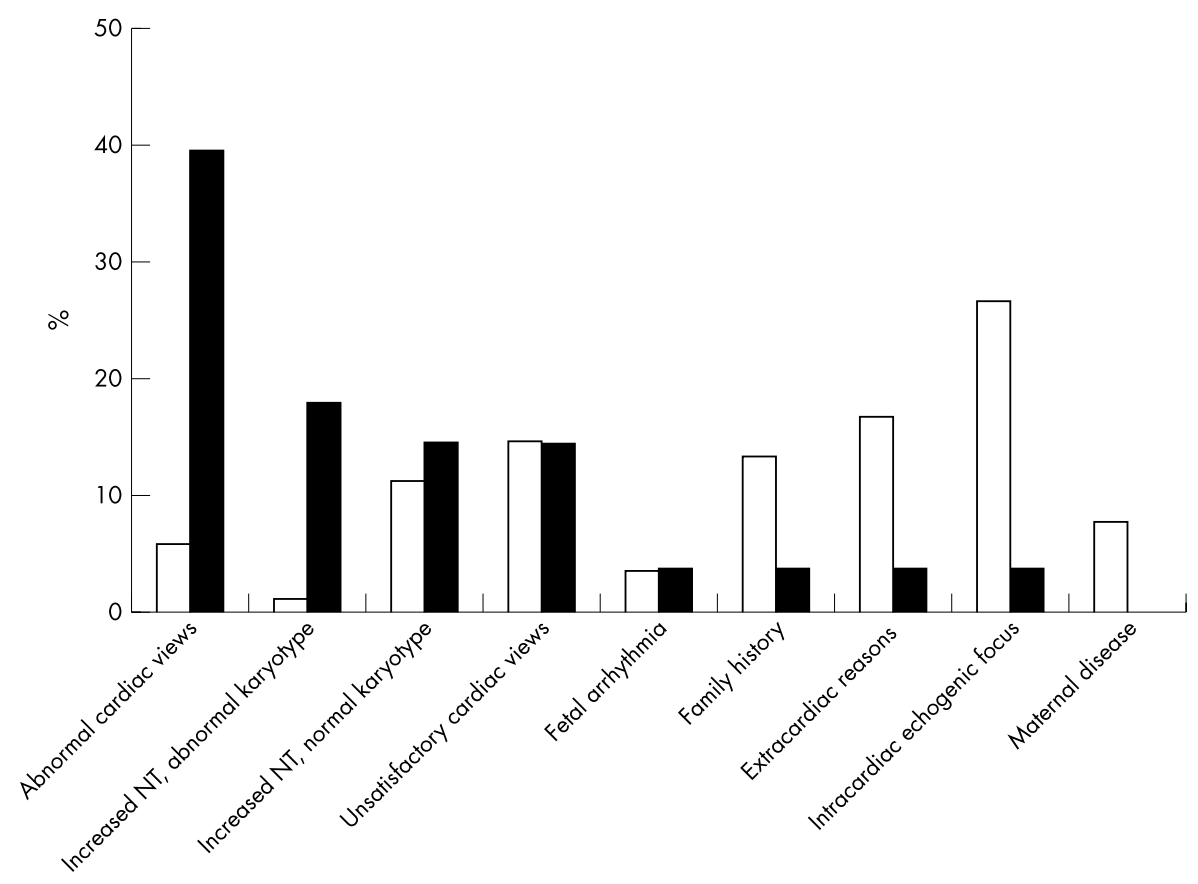

Figure 1 The frequency of referral for fetal echocardiography by indication (percentage of referred cases; open bars) and subsequent antenatal detection of major congenital heart defects (percentage of detected cases; solid bars). 
Table 1 Major cardiac defects: antenatal diagnoses in chromosomally normal and abnormal pregnancies

\begin{tabular}{|c|c|c|c|c|c|}
\hline $\begin{array}{l}\text { Case } \\
\text { number }\end{array}$ & Karyotype & $\begin{array}{l}\text { Time of } \\
\text { diagnosis } \\
\text { (weeks) }\end{array}$ & Indication for fetal echocardiography & Cardiac defect & Outcome \\
\hline 1 & Normal & 12 & Increased NT & Hypoplastic left heart syndrome & Termination at 12 weeks \\
\hline 2 & Normal & 12 & Fetal bradycardia & Left isomerism (complex) & Termination at 12 weeks \\
\hline 3 & Normal & 13 & Previous baby with $\mathrm{CHD}$ & $\begin{array}{l}\text { Severe aortic arch hypoplasia, } \\
\text { small left ventricle }\end{array}$ & Termination at 22 weeks \\
\hline 4 & Normal & 14 & Increased NT & Narrow aortic isthmus $(<0.4 \mathrm{~mm})$ & Termination at 14 weeks \\
\hline 5 & T21 & 14 & Increased NT & AVSD & Termination at 15 weeks \\
\hline 6 & T21 & 15 & Increased NT & AVSD & Intrauterine death at 17 weeks \\
\hline 7 & Normal & 17 & Increased NT, abnormal cardiac views & Hypoplastic left heart syndrome & Termination at 19 weeks \\
\hline 8 & Normal & 17 & Increased NT & Truncus arteriosus & Intrauterine death at 27 weeks \\
\hline 9 & $\mathrm{~T} 18$ & 18 & Increased NT & VSD, polyvalvar dysplasia & Intrauterine death at 37 weeks \\
\hline 10 & T13 & 20 & Abnormal cardiac views and increased NT & $\begin{array}{l}\text { Single outlet with pulmonary } \\
\text { atresia, aorta from RV }\end{array}$ & Neonatal death \\
\hline 11 & Normal & 20 & Abnormal cardiac views & Corrected TGA & Live birth, long term follow up \\
\hline 12 & Normal & 20 & Abnormal cardiac views & $\begin{array}{l}\text { Severe pulmonary stenosis/ } \\
\text { pulmonary atresia }\end{array}$ & $\begin{array}{l}\text { Preterm delivery at } 26 \text { weeks, } \\
\text { neonatal death }\end{array}$ \\
\hline 13 & Normal & 20 & Abnormal cardiac views & Tricuspid atresia & Termination at 21 weeks \\
\hline 14 & Normal & 20 & Abnormal cardiac views & Tetralogy of Fallot & Intrauterine death at 26 weeks \\
\hline 15 & Normal & 20 & Unsatisfactory cardiac views & Tetralogy of Fallot & Live birth, surgery \\
\hline 16 & T21 & 21 & Abnormal cardiac views and increased NT & AVSD & Live birth, surgery \\
\hline 17 & T18 & 21 & $\begin{array}{l}\text { Abnormal cardiac views - no fetal } \\
\text { echocardiography }\end{array}$ & Complex cardiac defect & Termination at 22 weeks \\
\hline 18 & $\mathrm{~T} 13$ & 21 & Abnormal cardiac views & Mitral atresia, double outlet RV & Termination at 22 weeks \\
\hline 19 & Normal & 21 & Abnormal cardiac views & Critical aortic stenosis & Termination at 22 weeks \\
\hline 20 & Normal & 21 & Abnormal cardiac views & Double inlet left ventricle & Live birth, surgery \\
\hline 21 & Normal & 22 & Unsatisfactory cardiac views & Aortic stenosis & Live birth, long term follow up \\
\hline 22 & Normal & 22 & Unsatisfactory cardiac views & Coarctation of aorta & Live birth, surgery \\
\hline 23 & Normal & 22 & Intracardiac echogenic focus & $\begin{array}{l}\text { Tetralogy of Fallot, pulmonary } \\
\text { atresia }\end{array}$ & Termination at 24 weeks \\
\hline 24 & Normal & 24 & Unsatisfactory cardiac views & $\begin{array}{l}\text { Corrected TGA, VSD, and } \\
\text { pulmonary atresia }\end{array}$ & Live birth, surgery \\
\hline 25 & Normal & 26 & Abnormal cardiac views - late booker & Atrioventricular septal defect & Live birth, surgery \\
\hline 26 & Normal & 27 & $\begin{array}{l}\text { Extracardiac reasons - severe growth } \\
\text { restriction }\end{array}$ & Situs inversus, complete TGA & $\begin{array}{l}\text { Preterm delivery at } 28 \text { weeks, } \\
\text { neonatal death }\end{array}$ \\
\hline 27 & Normal & 27 & Abnormal cardiac views on follow up scan & $\begin{array}{l}\text { Atrioventricular septal defect } \\
\text { primum ASD }\end{array}$ & Live birth, surgery \\
\hline 28 & T13 & 30 & Abnormal cardiac views - late booker & Small VSD, coarctation of aorta & Neonatal death \\
\hline 29 & $22 q$ deletion & 34 & Abnormal cardiac views on follow up scan & Truncus arteriosus & Live birth, surgery \\
\hline 30 & Normal & 39 & Abnormal cardiac views on follow up scan & Severe pulmonary stenosis & Live birth, balloon dilatation \\
\hline
\end{tabular}

for fetal echocardiography. During the study period, the most common reason for referral was the presence of intracardiac echogenic foci $(\mathrm{n}=235,26.4 \%)$ and the least frequent reason was the finding of abnormal cardiac views $(n=53,5.9 \%)$. The number of referrals for echocardiography fell from 26 per month (9.1/100 anomaly scans) at the beginning of the study to 23 per month (8/100 anomaly scans) at the end of the study.

\section{Major CHD}

Major defects of the heart and great arteries were identified in 40 pregnancies (4.3/1000 pregnancies) of which 30 were chromosomally normal (3.3/1000 pregnancies). Thirty of these cases $(75 \%)$ were diagnosed antenatally (table 1 ) and 10 postnatally (table 2 ). CHD were diagnosed in $70 \%$ of the chromosomally normal fetuses $(21 / 30)$ and in $90 \%$ of aneuploid fetuses (9/10; tables 1, 2, and 3). Most major CHD detected antenatally were diagnosed because of either abnormal or unsatisfactory cardiac views (21/30 or 70\%), the majority during scans carried out after 18 weeks (19/30 or 63\%), including two fetuses who also had increased nuchal translucency. Of these, 16 were suspected during the first anomaly scan and three on follow up examinations carried out for other reasons. Two were found to have abnormal cardiac findings before 18 weeks. One presented with fetal bradycardia during routine first trimester scan at 12 weeks (normal nuchal translucency) and in the other an abnormal four chamber view was detected at 17 weeks during a scan carried out for fetal viability because of maternal bleeding (previous nuchal translucency of $2.7 \mathrm{~mm}$ ).

Thus, abnormal or unsatisfactory cardiac views at any stage during pregnancy accounted for $70 \%$ of all prenatal diagnoses
(21/30) and for 53\% of all cases of CHD (21/40). After exclusion of those cases of CHD diagnosed in early pregnancy $(\leqslant 18$ weeks), the relative contribution of the sonographers's assessment of the fetal heart at the time of the routine anomaly scan ( $>18$ weeks) was $76 \%$ of all prenatal diagnoses (16/21) and $52 \%$ of all cases seen who had an anomaly scan after 18 weeks (16/31). Nine cases were diagnosed before the routine 18-23 week scan, seven of which were associated with increased nuchal translucency. Overall, the nuchal thickness was increased in 27\% of cases of CHD (9/34) and accounted for $15 \%(4 / 26)$ of chromosomally normal pregnancies where the mother underwent a scan at 12-14 weeks.

During the study period, 44 chromosomally abnormal pregnancies were diagnosed, of which 10 were diagnosed as having major CHD. In seven the defect was minor or absent. Twenty seven pregnancies were terminated before 14 weeks, before a fetal anomaly scan or fetal echocardiography was performed. A chromosomal abnormality was diagnosed because of increased nuchal translucency measurement in 32, because of multiple markers on anomaly scan in nine, and postnatally in the remaining three. Table 2 shows the indications for echocardiography in all cases diagnosed postnatally.

\section{DISCUSSION}

The present study has shown that routine prenatal screening for major CHD can be highly effective in a low risk population. The $75 \%$ detection rate of the current study is the highest reported with routine ultrasound screening, where ascertainment of the prevalence of CHD was thorough, including complete data on postnatal follow up. Although the data in large 


\begin{tabular}{|c|c|c|c|c|}
\hline $\begin{array}{l}\text { Case } \\
\text { number }\end{array}$ & Karyotype & Indication for echocardiography & Cardiac defect & Outcome \\
\hline 1 & Normal & Persistent cardiac murmur at 6 week follow up & VSD & Live birth, surgery \\
\hline 2 & Normal & Suspected VSD on antenatal scan & VSD & Live birth, surgery \\
\hline 3 & Normal & Central cyanosis and cardiac murmur & Pulmonary stenosis & Live birth, balloon dilatation \\
\hline 4 & Normal & Persistent cardiac murmur at 6 week follow up & Pulmonary stenosis & Live birth, balloon dilatation \\
\hline 5 & Normal & Central cyanosis and cardiac murmur & Tetralogy of Fallot & Live birth, surgery \\
\hline 6 & Normal & Tachypnoea and poor feeding & $\begin{array}{l}\text { Coarctation of the aorta, VSD, subaortic } \\
\text { stenosis }\end{array}$ & Live birth, surgery \\
\hline 7 & Normal & Neonatal collapse & $\begin{array}{l}\text { Coarctation of the aorta and bicuspid } \\
\text { aortic valve }\end{array}$ & Live birth, surgery \\
\hline 8 & Normal & Central cyanosis & Transposition of the great arteries & Live birth, surgery \\
\hline 9 & Normal & $\begin{array}{l}\text { Failure to thrive, cardiomegaly on chest } \\
\text { radiograph }\end{array}$ & Hypertrophic cardiomyopathy (LVOTO) & Live birth, long term follow up \\
\hline 10 & T21 & Postnatal diagnosis of T21 and cardiac murmur & VSD & Live birth, surgery \\
\hline
\end{tabular}

\begin{tabular}{|c|c|c|c|}
\hline & $\begin{array}{l}\text { Chromosomally } \\
\text { normal }(n=30)\end{array}$ & $\begin{array}{l}\text { Chromosomally } \\
\text { abnormal }(n=10)\end{array}$ & $\begin{array}{l}\text { Total pregnancies } \\
(\mathrm{n}=40)\end{array}$ \\
\hline \multicolumn{4}{|l|}{ Time of diagnosis (weeks) } \\
\hline$<18$ & 6 & $3 *$ & 9 \\
\hline $20-24$ & 11 & 4 & 15 \\
\hline $24-40$ & 4 & 2 & 6 \\
\hline Total antenatal detection rate (\%) & 70 & 90 & 75 \\
\hline $95 \%$ confidence interval & $51 \%$ to $85 \%$ & $56 \%$ to 100 & $59 \%$ to 87 \\
\hline
\end{tabular}

unselected obstetric populations were complete, the absolute number of abnormalities encountered is not large: 40 cases of major CHD, 30 of which were diagnosed antenatally. Thus, some caution should be exercised in extrapolating the detection rate to bigger populations. Our 95\% confidence interval, however, varied from $59 \%$ to $87 \%$.

\section{Prevalence of CHD and case ascertainment}

The overall prevalence for major CHD in this study was $4.3 / 1000$ pregnancies (3.3/1000 in chromosomally normal pregnancies). This figure is consistent with previous population reports on prevalence, indicating that ascertainment of fetal and infant CHD was rigorous in this study. ${ }^{3-5}$ Bull $^{14}$ recently conducted a multicentre study of 17 paediatric cardiac centres reporting 4799 major CHD in fetuses and infants delivered between 1993 and 1995. She reported a prevalence of 2.1/1000 pregnancies, although she noted that this may have been an underestimate because of underreporting of fetal diagnoses and necropsy results. Hunter and colleagues $^{17}$ reported prevalence rates between 2.6 and 3.3 /1000 live births between 1994 and 1997. Low prevalence of major CHD is a consistent problem in many previous studies, such that sensitivities as high as 55-63\% have been reported in studies where the CHD prevalence is as low as $1.3 / 1000$, presumably explained by the lack of adequate case ascertainment. ${ }^{11-13}$ Single centre studies, such as the current one, that included pathological examinations, neonatal echocardiography, and long term paediatric follow up have produced the most accurate estimates of CHD prevalence. Rustico and colleagues ${ }^{15}$ and Tegnander and associates ${ }^{8}$ used similar methods in large, prospective series and reported prevalences of 4.4 and 3.4/1000 pregnancies, respectively.

\section{Extended views and operator training}

Only two previous studies have evaluated the influence of sonographer training within the context of a routine screening programme. Tegnander and colleagues ${ }^{8}$ specifically investigated the value of operator training in a single unit with good long term follow up from a comprehensive child health programme. They reported an increase in the sensitivity from $18 \%$ to $26 \%$ with operator training to obtain a four chamber view. ${ }^{8}$ More recently, Hunter and colleagues ${ }^{17}$ carried out a four year prospective multicentre study to evaluate the effect of operator training on sensitivity of CHD screening. The authors reported an improvement in the detection rate for major CHD from $17 \%$ to $36 \%$ after a two year training period to enable sonographers to visualise the four chamber and outflow tract views. ${ }^{17}$ These data suggest that operator training and routine visualisation of the outflow tracts alone are unlikely to explain the sensitivity of $75 \%$ for CHD in this study.

\section{Indications for referral for detailed fetal echocardiography}

Approximately $20 \%$ of referrals were made because of an abnormal or unsatisfactory cardiac view at the routine fetal anomaly scan. Nonetheless, this resulted in the detection of $55 \%$ of major CHD. Although maternal diabetes and a family history of CHD also accounted for $20 \%$ of referrals, only one case $(3.3 \%)$ of major CHD was detected from these scans. The most common reason for referral $(26 \%)$ was the detection of isolated intracardiac echogenic foci, as dictated by the established protocol within the unit at the time of the study. The finding that the prevalence of major CHD in these fetuses appears to be no higher than in the rest of the population has now led to cessation of this practice. As fetuses with isolated intracardiac echogenic foci are no longer referred, approximately $5 \%$ of pregnancies now receive a detailed cardiac scan. It is important to note that increased nuchal translucency remains an important indication for fetal echocardiography, with $31 \%$ of major CHD diagnosed in this group. Although the sensitivity of this marker is lower than originally predicted, increased nuchal translucency above the 99th centile should mandate referral for detailed fetal echocardiography. ${ }^{24}$ 


\section{Examination time and access to echocardiography}

The reasons for the relatively high detection rate in this study deserve further analysis. Constraints placed on health service resources in the UK dictate that most routine fetal ultrasound examinations be performed in a limited amount of time and sometimes with inadequate equipment. In this study, sonographers were scheduled 20 minutes per fetal anomaly scan and they used ultrasound machines of a good standard, optimised for fetal cardiac examination.

The ease of access and the high frequency of referral in this study reflect the availability of fetal echocardiography within the same unit. The low threshold for fetal echocardiography and subsequent direct feedback enables sonographers gradually to build up their skills and confidence in detecting cardiac abnormalities. The sensitivity for CHD may have been relatively lower in the study carried out by Hunter and colleagues ${ }^{17}$ because it was based on data from 16 centres covering a large geographic area. The latter features mitigate against patient access and this is reflected by the finding that referrals for fetal echocardiography were low (1\%) compared with $8 \%$ in the current study. Few other studies of routine CHD screening have estimated the ease of access to and referral rate for fetal echocardiography following the anomaly scan, ${ }^{6-8}$ despite the obvious value of fetal cardiology services within a fetal medicine department.

\section{Conclusions}

Our data indicate that routine antenatal assessment of the four chambers and great vessels between 18 and 23 weeks is effective in the prenatal detection of major CHD. The sonographer performing the routine anomaly scan remains the major contributor to prenatal detection of major CHD. Proper equipment to allow optimisation of cardiac views, adequate examination time, and ease of access to tertiary level fetal cardiology services obviously influence the extent to which such a service is successful. While the need for improved antenatal detection of major CHD is known, the adoption of a screening programme similar to ours has significant resource implications for health care providers. Even a slight increase in scanning time may be more difficult to implement than training experienced sonographers to assess the ventricular outflow tracts and the four chamber view competently. Importantly, the success of this screening programme cannot be divorced from the ease of access and relatively high referral for detailed fetal echocardiography. The limited availability of such tertiary fetal cardiology specialists and services nationally is also likely to affect the success of other antenatal CHD screening programmes.

\section{ACKNOWLEDGEMENTS}

We thank the sonographers, midwives, and doctors in the Fetal Medicine Department at St George's Hospital, without whose contribution this work would not have been completed. Funding: EM was supported by a grant from St George's Hospital Trustees (grant 00.6.58). JSC was partially funded by the Hyman Marks Research Fund, Royal Brompton Hospital.

\section{Authors' affiliations}

*J S Carvalho, E Mavrides, S Campbell, B Thilaganathan, Fetal Medicine Unit, St George's Hospital Medical School, London, UK E A Shinebourne, Brompton Fetal and Paediatric Cardiology, Royal Brompton Hospital, London, UK

*Also Brompton Fetal and Paediatric Cardiology

\section{REFERENCES}

1 Tworetzky W, McEhlinney DB, Reddy V, et al. Improved surgical outcome after fetal diagnosis of hypoplastic left heart syndrome. Circulation 2001;103:1269-73.

2 Bonnet D, Coltri A, Butera G, et al. Detection of transposition of the great arteries in fetuses reduces neonatal morbidity and mortality. Circulation 1999;99:916-8.

3 Mitchell SC, Korones SB, Berendes HW. Congenital heart disease in 56,109 births: incidence and natural history. Circulation 1971:43:323-31

4 Hoffmann JIE, Christianson R. Congenital heart disease in a cohort 19,502 births with long-term follow up. Am J Cardiol 1978;42:641-7.

5 Ainsworth SB, Wyllie JP, Wren C. Prevalence and clinical significance of cardiac murmurs in neonates. Arch Dis Child 1999;80:F43-5.

6 Buskens E, Grobbee DE, Frohn-Mulder IM, et al. Efficacy of routine fetal ultrasound screening for congenital heart disease in normal pregnancy. Circulation 1996;1:67-72

7 Ott WJ. The accuracy of antenatal fetal echocardiography screening in high- and low- risk patients. Am J Obstet Gynecol 1995;172:1741-9.

8 Tegnander E, Eik-Nes SH, Johansen OJ, et al. Prenatal detection of heart defects at the routine fetal examination at 18 weeks in a non-selected population. Ultrasound Obstet Gynecol 1995;5:372-80.

9 Sharland GK, Allan LD. Screening for congenital heart disease prenatally: results of a $21 / 2$-year audit in the Southeast Thames Region. $\mathrm{Br}$ J Obstet Gynaecol 1992;9:220-5.

10 Vergani P, Mariani S, Ghidini A, et al. Screening for congenital heart disease with the four-chamber view of the fetal heart. Am J Obstet Gynecol 1992;167:1000-4.

11 Luck CA. Value of routine ultrasound scanning at 19 weeks: a four-year study of 8849 deliveries. BM 1992:304:1474-8.

12 Shirley IM, Bottomley F, Robinson P. Routine radiographer screening for fetal abnormalities by ultrasound in an unselected low risk population. $\mathrm{Br}$ J Radiol 1992:65:564-9.

13 Chitty LS, Hunt GH, Moore J, et al. Effectiveness of routine ultrasonography in detecting fetal structural abnormalities in a low risk population. BN 1991;303:1165-9.

14 Bull C. Current and potential impact of fetal diagnosis on prevalence and spectrum of serious congenital heart disease at term in the UK. Lancet 1999;354: 1242-7

15 Rustico MA, Benettoni A, D'Ottavio GD, et al. Fetal heart screening in low-risk population. Ultrasound Obstet Gynecol 1995;6:313-9.

16 Achiron R, Glaser J, Gelernter I, et al. Extended fetal echocardiographic examination for detecting cardiac malformations in low risk pregnancies. BM 1992;304:671-4.

17 Hunter S, Heads A, Wyllie J, et al. Prenatal diagnosis of congenital heart disease in the northern region of England: benefits of a training programme for obstetric ultrasonographers. Heart 2000;84:294-8.

18 Snijders RJ, Noble P, Sebire N, et al. UK multicentre project assessment of risk of trisomy 21 by maternal age and fetal nuchal translucency thickness at 10 to 14 weeks of gestation. Fetal Medicine Foundation First Trimester Screening Group. Lancet 1998;352:343-6.

19 Meyer-Wittkopf M, Simpson JM, Sharland GK. Incidence of congenital heart defects in fetuses of diabetic mothers: a retrospective study of 326 cases. Ultrasound Obstet Gynecol 1996;8:8-10.

20 Rose V, Gold RJM, Lindsey G, et al. A possible increase in the incidence of congenital heart defects among the offspring of affected parents. J Am Coll Cardiol 1985;6:376-82.

21 Allan LD, Crawford DC, Chita SK, et al. Familial recurrence of congenital heart disease in a prospective series of mothers referred for fetal echocardiography. Am J Cardiol 1986;58:334-7.

22 Burn J, Brennan P, Little J. Reccurence risks in offspring of adults with major heart defects: results from first cohort of British collaborative study. Lancet 1998;351:311-6.

23 Zierler S. Maternal drugs and congenital heart disease. Obstet Gynecol 1985:5:155-9

24 Hyett J, Perdu M, Sharland G, et al. Using fetal nuchal translucency to screen for major congenital defects at 10-14 weeks of gestation: population based cohort study. BM 1999:318:81-5.

25 Mavrides E, Cobian-Sanchez F, Moscoso G, et al. Limitations of first trimester nuchal translucency measurement in screening for major congenital heart defects. Ultrasound Obstet Gynecol 2001;17:106-10.

26 Zosmer N, Souter VL, Chan CSY, et al. Early diagnosis of major cardiac defects in chromosomally normal fetuses with increased nuchal translucency. Br J Obstet Gynaecol 1999;106:829-33.

27 Carvalho JS, Moscoso G, Ville Y. First-trimester transabdominal fetal echocardiography. Lancet 1998;351:1023-7. 